\title{
Comment on: "Impact of portable normothermic blood-based machine perfusion on outcomes of liver transplant: the OCS Liver PROTECT randomized clinical trial"
}

\author{
Arthur Marichez, Jean-Philippe Adam \\ Department of Hepato-Bilio-Pancreatic Surgery and Liver Transplantation, Haut Lévêque Hospital, CHU de Bordeaux, France \\ Correspondence to: Arthur Marichez. Department of Hepato-Bilio-Pancreatic Surgery and Liver Transplantation, Haut Lévêque Hospital, CHU de \\ Bordeaux, France. Email: arthur.marichez@chu-bordeaux.fr. \\ Comment on: Markmann JF, Abouljoud MS, Ghobrial RM, et al. Impact of Portable Normothermic Blood-Based Machine Perfusion on Outcomes of \\ Liver Transplant: The OCS Liver PROTECT Randomized Clinical Trial. JAMA Surg 2022. [Epub ahead of print]. doi:10.1001/jamasurg.2021.6781.
}

Submitted Jan 18, 2022. Accepted for publication Jan 30, 2022.

doi: $10.21037 / \mathrm{hbsn}-22-25$

View this article at: https://dx.doi.org/10.21037/hbsn-22-25

We read with great interest the paper by Markmann et al. entitled "Impact of portable normothermic blood-based machine perfusion on outcomes of liver transplant: The OCS Liver PROTECT randomized clinical trial" (1). In this multicenter randomized clinical trial, the authors compared posttransplant outcomes for recipients who received donation after brainstem death (DBD) or donation after circulatory death (DCD) livers preserved using ischemic cold storage (ICS) or a portable normothermic machine perfusion (NMP) [Organ Care System (OCS) Liver]. The primary endpoint was the incidence of early allograft dysfunction (EAD) as defined by Olthoff et al. criteria (2). The trial was methodologically designed to test both noninferiority on the primary endpoint, and superiority if noninferiority was achieved. This study was conducted from 2016 to 2019 at 20 US liver transplant programs. From the 476 patients randomized after graft acceptance and before the harvesting team left, 300 recipients were included: 153 patients in the OCS group and 147 in the ICS group. Both groups were comparable, except for the proportion of DCD grafts, which was twice as high in the OCS group compared to the ICS control group (19\% vs. 9\%). The trial met its primary efficacy endpoint by demonstrating statistical noninferiority and superiority of outcomes for the OCS group compared to the ICS group in the per-protocol population and also in the intention-to-treat analysis population. The incidence of EAD was significantly reduced in the OCS group compared to the ICS group in the per-protocol analysis ( $18 \%$ vs. $31 \%$, respectively; $\mathrm{P}=0.01$ ), as well as in the intention-to-treat analysis with a relative decrease in EAD of $45 \%$ in the OCS group $(\mathrm{P}=0.005)$. Regarding the secondary endpoints, ischemia-reperfusion injury $(6 \% \mathrm{vs}$. $13 \% ; \mathrm{P}=0.004)$, and the rate of non-anastomotic ischemic biliary complications at 6 months $(1.3 \%$ vs. $8.5 \% ; \mathrm{P}=0.02)$ and at 12 months $(2.6 \%$ vs. $9.9 \% ; \mathrm{P}=0.02)$ were significantly reduced in the OCS group, while 30 -day patient survival was comparable between the 2 groups.

This is the first multicenter randomized controlled trial to test a portable NMP directly used at the donor hospital. Given the complexity of both the logistics and the normothermic perfusion process, the authors are to be congratulated on their success in conducting this trial, which included a substantial number of patients in a limited period of time at 20 US centers. Their important findings were awarded at the American Congress of Transplantation, and subsequently allowed the OCS Liver machine to be granted pre-market approval by the FDA (3). The OCS Liver machine is now the only FDA-approved ex vivo perfusion system for prolonging the viability of donor livers for transplantation.

This trial provides some new interesting data regarding normothermic perfusion of liver grafts compared to previous published randomized clinical trials $(4,5)$.

Firstly, the authors chose EAD defined according to the Olthoff's criteria as primary endpoint. This is a more relevant clinical criterion for assessing the performance of the machine than the peak level of transaminases, as used in the trial published by Nasralla et al. (4). Although the 
authors did not detail and explain the calculation of the sample size in order to validate the statistical hypothesis, they found that normothermic perfusion on the OCS Liver machine reduced EAD by nearly $45 \%$ compared with ICS. As already reported in the literature, EAD was associated in this trial with a significant reduction in the length of ICU stay, and with a worse graft survival at 12 months. However, at 12 months after transplantation, the use of the OCS liver did not affect graft and patient survival which were comparable with the ICS group, despite a significant decrease in the rate of ischemic biliary complications after normothermic perfusion ( $2.6 \%$ vs. $9.9 \%)$.

The main innovative aspect of this study concerns the modality of the normothermic perfusion of the liver graft, related to the portability of the machine. Despite a logically longer total preservation time in the OCS group, the mean duration of cold ischemia was significantly reduced, by almost $3 \mathrm{~h}$ compared to the ICS group (175 vs. 339 min, $\mathrm{P}<0.001)$. In this trial, NMP was started directly at the hospital donor, without prior cold ischemic storage. In previously published studies, it was a post-cold storage NMP with a mean duration of cold ischemic storage ranging from 2 to 8 hours (4-6). The authors highlight the importance of avoiding this duration of cold ischemia to optimize outcomes, particularly for DCD grafts for which the incidence of EAD can reach up to $46 \%$ (7). In France, the incidence of EAD for DCD grafts is around $18 \%$ and similar to the OCS group, thanks to both the application of selection criteria for DCD grafts and the use of in situ normothermic regional perfusion (8).

Another advantage of the OCS machine highlighted by the authors was the increase in the number of DCD grafts used compared to the control group (51\% vs. $26 \%$; $\mathrm{P}=0.007)$. This imbalance between both groups could be explained by the unblinded process of randomization, as the authors explained. For obvious logistical reasons related to the transport of the machine, randomization was performed before the departure of the harvesting team, potentially resulting in a stricter selection of grafts in the group without machine. This probably also explains the higher number of discarded grafts during assessment in the ICS group compared to the OCS group (22 vs. 9, respectively). Despite this potential selection bias, the use of the machine probably allowed more grafts to be accepted and the outcomes were not adversely affected by the higher proportion of DCD grafts in the OCS group.

Concerning the feasibility of the procedure, only 3 minor problems (2\%) were reported, without any consequence on the success of the transplantation. However, the lack of data on the transport of the perfused graft is regrettable. Given the complexity of perfusion in normothermic conditions and the logistics involved in transporting the machine, it would have been interesting to know how the machine was transported and monitored from the donor hospital site to the transplant center, the duration of transport, and what the learning curve was for its use.

In conclusion, The OCS Liver PROTECT trial is the first randomized controlled trial to demonstrate the reduction of early graft dysfunction by the use of a portable normothermic perfusion machine without prior ICS. The trial adds to previous randomized trials of normothermic $(4,5)$ and hypothermic $(9,10)$ perfusion machine and supports the benefit of machines in expanding the donor pool and in optimizing the outcomes of liver transplantation. Data are still needed to specify the complementarity of normothermic and hypothermic perfusion machines.

\section{Acknowledgments}

Funding: None.

\section{Footnote}

Provenance and Peer Review: This article was commissioned by the editorial office, Hepatobiliary Surgery and Nutrition. The article did not undergo external peer review.

Conflicts of Interest: Both authors have completed the ICMJE uniform disclosure form (available at https://hbsn. amegroups.com/article/view/10.21037/hbsn-22-25/coif). The authors have no conflicts of interest to declare.

Ethical Statement: The authors are accountable for all aspects of the work in ensuring that questions related to the accuracy or integrity of any part of the work are appropriately investigated and resolved.

Open Access Statement: This is an Open Access article distributed in accordance with the Creative Commons Attribution-NonCommercial-NoDerivs 4.0 International License (CC BY-NC-ND 4.0), which permits the noncommercial replication and distribution of the article with the strict proviso that no changes or edits are made and the original work is properly cited (including links to both the formal publication through the relevant DOI and the license). 
See: https://creativecommons.org/licenses/by-nc-nd/4.0/.

\section{References}

1. Markmann JF, Abouljoud MS, Ghobrial RM, et al. Impact of Portable Normothermic Blood-Based Machine Perfusion on Outcomes of Liver Transplant: The OCS Liver PROTECT Randomized Clinical Trial. JAMA Surg 2022. [Epub ahead of print]. doi:10.1001/ jamasurg.2021.6781.

2. Olthoff KM, Kulik L, Samstein B, et al. Validation of a current definition of early allograft dysfunction in liver transplant recipients and analysis of risk factors. Liver Transpl 2010;16:943-9.

3. Chang DD, Han JJ. The TransMedics Organ Care System for the liver receives FDA pre-market approval. Artif Organs 2022;46:25-6.

4. Nasralla D, Coussios CC, Mergental H, et al. A randomized trial of normothermic preservation in liver transplantation. Nature 2018;557:50-6.

5. Ghinolfi D, Rreka E, De Tata V, et al. Pilot, Open, Randomized, Prospective Trial for Normothermic Machine Perfusion Evaluation in Liver Transplantation From Older Donors. Liver Transpl 2019;25:436-49.

Cite this article as: Marichez A, Adam JP. Comment on: "Impact of portable normothermic blood-based machine perfusion on outcomes of liver transplant: the OCS Liver PROTECT randomized clinical trial". HepatoBiliary Surg Nutr 2022;11(2):267-269. doi: 10.21037/hbsn-22-25
6. Mergental H, Laing RW, Kirkham AJ, et al. Transplantation of discarded livers following viability testing with normothermic machine perfusion. Nat Commun 2020;11:2939.

7. Wang K, Lu D, Liu Y, et al. Severity of early allograft dysfunction following donation after circulatory death liver transplantation: a multicentre study. Hepatobiliary Surg Nutr 2021;10:9-19.

8. Savier E, Lim C, Rayar M, et al. Favorable Outcomes of Liver Transplantation from Controlled Circulatory Death Donors Using Normothermic Regional Perfusion Compared to Brain Death Donors. Transplantation 2020;104:1943-51.

9. van Rijn R, Schurink IJ, de Vries Y, et al. Hypothermic Machine Perfusion in Liver Transplantation - A Randomized Trial. N Engl J Med 2021;384:1391-401.

10. Czigany Z, Pratschke J, Froněk J, et al. Hypothermic Oxygenated Machine Perfusion Reduces Early Allograft Injury and Improves Post-transplant Outcomes in Extended Criteria Donation Liver Transplantation From Donation After Brain Death: Results From a Multicenter Randomized Controlled Trial (HOPE ECD-DBD). Ann Surg 2021;274:705-12. 